\section{GENETIC RADIATION HAZARDS}

\section{Radiation, Genes, and Man}

By Prof. Bruce Wallace and Prof. Th. Dobzhansky. Pp. xii +205 . (New York : Henry Holt and Company, Inc., 1959.) 3.50 dollars.

A

FTER an initial free-for-all, in which soothing or alarmist prophecies were made on a minimum of evidence, the subject of radiation hazards is now settling down and accumulating solid facts, as a basis for firmer predictions. So solid, indeed, are some of the facts released by recent work on the genetic effects of radiation that several cherished beliefs lie smashed or at least splintered. At such a time, when the real complexity of the subject is just beginning to loom ahead, it is best to tread with caution and avoid dogmatic statements, especially when writing a book. For bold generalizations may be quickly overthrown, if one is unlucky. This otherwise valuable book has suffered from such a process.

"Radiation, Genes and Man" has the laudable aim of presenting the relevant facts on which the reader himself can judge the magnitude of genetic radiation hazards and can also critically assess the pronouncements of others on the subject. In order to do this, the authors have had to cover a very wide field, ranging from elementary radiation physies to fairly advanced population genetics. On the latter they speak with authority and many will find it the most useful part of the book. But in some other fields there is a tendency towards over-simplification, which may prove misleading. For example, the section on meiosis describes and illustrates it as if no recombination takes place. The use of blue eyes versus brown as an example of simple Mendelian segregation in man has its dangers too, as there are enough exceptions and complications to make some people unnecessarily puzzled, if not suspicious.

Until recently, radiation genetics was mainly concerned with the results of irradiating postmeiotic germ-cells of Drosophila. In man, however, as the authors point out, effects of radiation on spermatogonia and oocytes are more important; thus most work on the mouse has been concerned with these stages. Unfortunately the authors tend to ignore the fact that results obtained from irradiating sperm which have finished dividing need not apply to actively dividing cells such as spermatogonia, in which selective forces are different. They declare, for example, that neither dose-rate nor fractionation of dose has any effect on induced mutation frequency. Yet it is now firmly established (thanks mainly to the work of Russell and collabora. tors at Oak Ridge) that acute X-rays have a significantly higher mutagenic effect on mouse spermatogonia and oocytes than the same dose of chronic gamma-rays, though the quality of the radiation (X versus gamma) seems unimportant. First results of this work are mentioned twice by the authors but once, unfortunately, 'crossing-over' has occurred, figures of induced mutation frequency for acute and chronic radiation being given the wrong way round (p. 131).

It is now known, too, that fractionation of dose may have a marked effect in certain circumstances. Evidence on all these matters is of great interest to man, for if lower dose-rates are less mutagenic for us, then genetic hazards from medical radiology become relatively more important than previously thought, while those from an increase in background radiation due to radioactive fall-out, etc., become relatively less important. It also means that we can no longer be content with the authors' simple definition of doubling dose as "the dose of radiation required to induce the same amount of gene mutation as that which arises spontaneously without radiation". We can now see that the answer will depend on germcell stage irradiated, period of time over which the dose was given, fractionation perhaps and possibly also the sex of the animal and whether irradiated as a foetus. Thus the figure is rapidly being replaced by a formula.

Despite these criticisms, the book as a whole can be recommended, for it gives a useful introduction to theoretical aspects of radiation genetics and the problems which must be solved before we have any accurate measure of the extra genetic load imposed by ionizing radiations. The authors stress the need for further research, especially into the behaviour of mutants in populations, so that we may learn more about such important factors as the extent to which mutant heterozygotes are more or less fit than normal. In their concluding survey they rightly point out the need for "value judgments" on procedures exposing persons to radiation, in which the benefits expected are weighed against the disadvantages. Using these criteria, they conclude that nuclear bomb tests should stop. The book ends with an all-too-short glossary and a bibliography.

A. G. Searle

\section{THE NEW COSMOGRAPHY}

\section{The Inner Metagalaxy}

By Prof. Harlow Shapley. Pp. xiii +204. (London : Oxford University Press ; New Haven, Conn. : Yale University Press, 1957.) 55s. net.

$\mathrm{W}$ HEN the history of astronomy in the twentieth century comes to be written, neither the recent developments in radio astronomy nor in satellite research will hold the primary place. The most significant discovery of the century (no matter what is yet to come) has been the recognition, in the period from 1915 to 1925 , that we are inhabitants of no mean universe : the Sun was then finally relegated to its now accepted place, "an awkward position near the edge of one dusty spiral", as Prof. Shapley puts it, and the Galaxy itself was seen to be but one of millions more, scattered through space as far as our largest telescope can reach.

The complexity of modern science, and the temporal proximity of the event, do not enable us to attribute to a single twentieth century Copernicus the discovery of the new "system of the world". But the author of this book is one of a very few astronomers who brought about the greatest of all revolutions in our understanding of the universe into which we are born. It was Prof. Shapley's study of the distribution of the globular clusters that first pointed accurately the direction in which the galactic centre lay, and in the decades that followed he and his colleagues at the Harvard College Observatory sketched in, with bold strokes of the brush, the essential outlines of this larger universe which will not be radically altered. The patient counting and sampling of the millions of galaxies recorded on photographs taken at the various Harvard out. 